\title{
A Philosophical Look at the Higgs Mechanism
}

\author{
Simon Friederich \\ email@simonfriederich.eu \\ Universität Göttingen, Philosophisches Seminar, Humboldtallee 19, \\ D-37073, Germany ${ }^{1}$
}

\begin{abstract}
On the occasion of the recent experimental detection of a Higgstype particle at the Large Hadron Collider (LHC) at CERN, the paper reviews philosophical aspects of the Higgs mechanism as the presently preferred account of the generation of particle masses in the Standard Model of elementary particle physics and its most discussed extensions. The paper serves a twofold purpose: on the one hand, it offers an introduction to the Higgs mechanism and its most interesting philosophical aspects to readers not familiar with it; on the other hand, it clarifies widespread misunderstandings related to the role of gauge symmetries and their breaking in it.
\end{abstract}

\section{Introduction}

The Higgs mechanism is a crucial ingredient of the Standard Model of elementary particle physics. Its role is to reconcile the experimentally determined short range of one of the fundamental forces of nature, the weak force (which manifests itself, for instance, in radioactive beta decay), with the theoretical requirement of renormalisability (roughly meaning that the theory remains predictive when applied to the description of processes at high energies and short distances).

The simplest way of setting up the basic idea of the Higgs mechanism involves the so-called Higgs particle. In this picture, the interactions between the Higgs particle and the particles mediating the weak force give rise to the masses of the latter, thus accounting for the short range of the weak force, as determined experimentally. In the full-fledged implementation of the Higgs mechanism the Higgs particle couples not only to those particles which mediate forces between matter particles but also to the matter particles themselves, such that it generates their non-vanishing masses as

\footnotetext{
${ }^{1}$ Work carried out at the universities of Wuppertal and Cambridge UK
} 
well. Recent experimental findings at the Large Hadron Collider (LHC) in Geneva at CERN have been interpreted as direct detection of a particle with properties as expected for the Higgs particle, which is widely regarded as spectacular confirmation of this picture. On occasion of these experiments, the present contribution gives an introduction to philosophically challenging aspects of the Higgs mechanism, in particular by clarifying the role and status of fundamental symmetries of nature and their so-called "spontaneous breaking" in it.

The symmetries used to formulate the theories of the fundamental interactions combined in the Standard Model are gauge symmetries or, more precisely, local gauge symmetries, meaning that the symmetry transformations associated with these symmetries depend on infinitely many variables labelled by the ("local") points of space-time. Local symmetries contrast with global (i. e. spacetime-independent) ones, where symmetry transformations relating configurations depend only on a single parameter that can be seen as constant throughout space-time and as in that sense "global". Configurations of the gauge fields that can be transformed into each other by gauge transformations, at least on the common view of gauge symmetries, represent one and the same physical situation. According to the textbook picture of the Higgs mechanism (to be clarified and slightly refined in this article), the generation of particle masses through the Higgs mechanism is made possible by the "spontaneous breaking" of a local gauge symmetry.

The basic idea behind the notion of spontaneous symmetry breaking ("SSB"), to be made slightly more precise in Section 3, is that a symmetry of the laws of motion of a system may be radically absent from some of the solutions to these equations in the sense that it connects field configurations that cannot be reached from each other by means of "physically realizable operations." ${ }^{2}$ The suggestion that the spontaneous breaking of a gauge symmetry should be at the origin of the generation of particle masses may seem astounding in view of the received view that gauge symmetries are not empirical symmetries, that is, that they do not themselves correspond to empirical features of physical reality, so that one may wonder what it could mean for such a symmetry to be spontaneously broken at all. As put by Chris Smeenk, "[i]f gauge symmetry merely indicates descriptive redundancy in the mathematical formalism, it is not clear how spontaneously breaking a gauge symmetry could have any physical consequences, desirable or not." 3

Thus, the aim of the present paper is, on the one hand, to provide a

\footnotetext{
${ }^{2}$ See [Strocchi, 2008] p. 4.

${ }^{3}$ See [Smeenk, 2006] p. 488.
} 
short introduction to the motivation and main idea of the Higgs mechanism and, on the other, to dissolve the puzzlement expressed in the (rhetorical) statement by Smeenk and, thereby, elucidate the role of gauge symmetries and their breaking in it.

The rest of the paper is structured as follows: Section 2 provides a concise introduction to gauge theories. Section 3 sketches the problem the Higgs mechanism is supposed to solve, namely, the difficulty of theoretically accounting for the experimentally observed particle masses in the context of the Standard Model of elementary particle physics. Section 4 recapitulates the textbook classical argument of how particle masses are generated in the Higgs mechanism through their interaction with the Higgs particle, before Section 5 widens the scope to include considerations involving the quantum nature of the particles described by the gauge theories in the Standard Model. By appeal to an important result known as Elitzur's theorem, it is argued that, contrary to widespread views, the Higgs mechanism should not be spelled out in terms of the notion of a spontaneously broken local gauge symmetry in the quantum context. In addition, the actual role of spontaneously broken gauge symmetries is elucidated in more detail by discussing the status of the breaking of remnant global gauge symmetries. These are symmetries under which gauge theories are typically invariant after a procedure called "fixing the gauge", a procedure which helps to make gauge theories well-defined when written in terms of the space-time continuum but violates local gauge invariance. The paper closes in Section 6 with a brief summary.

\section{Conceptual basics}

The theories of the fundamental interactions of nature (except gravity), which are combined in the Standard Model of elementary particle physics, belong to a class of physical theories known as "gauge theories". The defining mathematical characteristic of these theories is that they are defined in terms of an action $S$ that is invariant with respect to the elements of a continuous group of local transformations. The invariance of the action under these mappings is referred to as "gauge symmetry", and the mappings themselves are called "gauge transformations". (Examples of such symmetries will be given and discussed below.)

As follows from Noether's second theorem (see [Noether, 1918]), the equations of motion give rise to an apparent failure of determinism in this 
case in that their solutions involve arbitrary functions of time. ${ }^{4}$ In the physical interpretation of gauge theories, however, determinism can be preserved by assuming that configurations that are related by the symmetry represent identical physical situations. In a gauge theory, in other words, each physically distinct situation is represented in terms of an infinite class of mathematically distinct variable configurations related by gauge transformations. Only those combinations of variables that do not change when gauge transformations are performed (the "gauge invariant" ones) can be physical quantities.

The standard perspective on gauge symmetries as non-empirical in the sense that different configurations of gauge fields related by gauge transformations correspond to the same physical situation has not gone uncontested among philosophers. It is sometimes criticised for having difficulties to account for a class of apparently non-local phenomena in quantum theory such as the famous Aharonov-Bohm effect. ${ }^{5}$ But another often-heard criticism is that gauge symmetry breaking - allegedly a hallmark of the Higgs mechanism, discussed in this paper-warrants an interpretation of gauge symmetries as having a more direct empirical significance. ${ }^{6}$ At the end of Section 5, I will critically address these claims in the light of the considerations on gauge symmetry breaking, to be presented in the meantime. It remains possible, however, to interpret gauge symmetries as representing features of physical reality in a more indirect way. An account which does so is Lyre's structural realist perspective on gauge theories, where gauge symmetries are regarded as manifesting themselves in the invariants under gauge transformations, conceived of as "structurally derived intrinsic properties." 7 None of the considerations to be presented in what follows seem to be in conflict with the claims made by such an approach.

The remaining part of this section introduces the basic notions of gauge theories and gauge symmetries in a more concrete, but also more technical,

\footnotetext{
${ }^{4}$ See, for instance, [Earman, 2004], Section 6, and [Struyve, 2011], Section 7, for more detailed discussions of this point, which are aimed at philosophers.

${ }^{5}$ The standard view of gauge symmetries as non-empirical is attacked by [Maudlin, 1998] in terms of considerations on the Aharonov-Bohm effect, a direct response by Healey defending the standard view can be found in [Healey, 1998]; a more recent defence of the standard view of gauge symmetries as non-empirical and a careful assessment of its philosophical ramifications can be found in [Healey, 2007] and [Healey, 2009]. For a recent criticism of the standard perspective on local gauge symmetries as in all circumstances non-empirical see [Greaves and Wallace, 2011].

${ }^{6}$ See, for instance, [Weinberg, 1974].

${ }^{7}$ See [Lyre, 2010]. For further interesting considerations on the prospects of structural realist readings of gauge symmetries see [Roberts, 2011].
} 
language. Readers familiar with gauge theoretical notions may skip the following paragraphs without loss.

Electromagnetism is a prime example of a gauge theory. To discuss its formulation in terms of gauge fields, consider first the Lagrangean (the difference $\mathcal{L}=T-V$ between the kinetic and potential energy densities $T$ and $V$ ), which allows one to derive the equations of motion for the electric and magnetic fields:

$$
\mathcal{L}=-\frac{1}{4} F_{\mu \nu} F^{\mu \nu}
$$

where summation over doubly occurring indices $\mu, \nu=0,1,2$, 3, labelling the four dimensions of space-time, is understood, as in everything that follows. The mathematical object $F_{\mu \nu}$ is the relativistic electromagnetic field tensor. Its six independent entries are the spatial components of the electric and magnetic fields $\mathbf{E}$ and $\mathbf{B} .^{8}$ In the formulation of electromagnetism as a gauge theory, the gauge fields come in the form of the vector potential $A_{\mu}=(\phi,-\mathbf{A})$, which encodes all information about the electric and magnetic fields in that these are obtained from $A_{\mu}$ by taking derivatives: $\mathbf{E}=-\nabla \phi-\partial_{t} \mathbf{A}$ and $\mathbf{B}=\operatorname{rot} \mathbf{A}$. The gauge transformations which leave the Lagrangean invariant are given by

$$
A_{\mu}(x) \mapsto A_{\mu}(x)+\partial_{\mu} \Lambda(x),
$$

where $\Lambda(x)$ is an arbitrary function of space-time variables. The transformations of the form Eq. (2) are called local gauge transformations, where the attribute "local" refers to the fact that the transformations depend on infinitely many parameters corresponding to the ("local") points of spacetime. The electromagnetic tensor $F_{\mu \nu}$ can be expressed in terms of the field $A_{\mu}$ as

$$
F_{\mu \nu}=\partial_{\mu} A_{\nu}-\partial_{\nu} A_{\mu} .
$$

As required, the tensor $F_{\mu \nu}$ is invariant under the gauge transformations Eq. (2), which means that the same also holds for its entries, the components of the electric and magnetic fields. This means that configurations of the field $A_{\mu}(x)$ that are taken to each other by gauge transformations of the form Eq. (2) describe the same electric and magnetic fields $\mathbf{E}$ and $\mathbf{B}$, which is naturally taken to suggest that they correspond to the same physical situation.

\footnotetext{
${ }^{8}$ See Chapter 11.9 of [Jackson, 1998] for a canonical textbook introduction to the relativistic formulation of electromagnetism, where the tensor $F_{\mu \nu}$ is introduced and its properties are discussed in great detail.
} 
To be able to describe electromagnetic interactions involving particles, one needs to include matter fields $\psi$ in the Lagrangean (2). Just as the matter fields correspond to matter particles in the quantised theory, the gauge fields such as $A_{\mu}$ correspond to the so-called "gauge particles", also referred to as "gauge bosons". As it turns out, the role of the gauge particles can be seen as that of mediators of the interactions between the matter particles. A simple example of a gauge theory describing matter particles and their interactions as mediated by gauge particles is quantum electrodynamics ("QED"), the quantised theory of electromagnetic interactions involving electrons, positrons (the opposite-charged twins of the electrons) and the light quanta known as photons. The Lagrangean of this theory is given by

$$
\mathcal{L}=-\frac{1}{4} F_{\mu \nu} F^{\mu \nu}+\bar{\psi}\left(i \gamma^{\mu} D_{\mu}-m\right) \psi
$$

where $i=\sqrt{-1}$ denotes the imaginary unit, $\psi$ denotes the electron field and $\bar{\psi}$ denotes the positron field. The matrices $\gamma^{\mu}$ are the so-called $\gamma$ - or Dirac matrices, defined by the relation $\gamma^{\mu} \gamma^{\nu}+\gamma^{\nu} \gamma^{\mu}=2 \eta^{\mu \nu} \mathbf{1}$, where $\eta^{\mu \nu}$ is the space-time metric matrix which has nonzero entries $\{1,-1,-1,-1\}$ only on its diagonal. The parameter $m$ denotes the electron mass and $D_{\mu}$ is the covariant derivative defined by

$$
D_{\mu}=\partial_{\mu}+i e A_{\mu}
$$

where $e$ is the electron charge. The interaction between the matter and gauge fields is expressed in Eq. (4) by the term which includes the covariant derivative.

In order to be able to describe not only the electromagnetic interaction but also the weak and strong interactions between elementary particles, the gauge theories under consideration must be generalised to so-called non-abelian gauge theories. In non-abelian gauge theories, the gauge transformations with respect to which the Lagrangean is invariant no longer in general commute with each other, which means that the outcome of consecutively performing two such transformations depends on the order in which they are carried out. Mathematically speaking, the symmetry transformations in a gauge theory form a symmetry group, the elements of which may either commute with each other (abelian case) or not (non-abelian case).

The mathematical groups used to define the gauge symmetries are Lie groups, and any Lie group element $U$ can be expressed in terms of certain group elements called the generators $T_{a}$ of the group together with real parameters $\theta^{a}$ as

$$
U=\exp \left(i g \theta^{a} T_{a}\right)
$$


where $a$ ranges over $\{1, \ldots, N\}$ with $N$ denoting the dimension of the group (the number of independent parameters necessary to specify a group element).

The non-commutative structure of a non-abelian Lie group is encoded in the commutation relations for the generators, which can be written in the form

$$
T_{a} T_{b}-T_{b} T_{a}=i g f_{a b}^{c} T_{c}
$$

with summation over the doubly occurring index $c$.

In the context of a gauge theory, the parameter $g$, called the "gauge coupling", measures the strength of the interaction mediated by the gauge particles. Its role is like that of the electric charge $e$ in the case of quantum electrodynamics. The numbers $f_{b c}^{a}$ are the structure constants of the Lie group, which, figuratively speaking, measure the degree of non-commutativity of the group. Examples of Lie groups that are often used in gauge theories include the group of rotations in $N$-dimensional space (denoted $S O(N)$ ) and the group of unitary $N \times N$-matrices with complex entries and determinant +1 (denoted $S U(N)$ ). A simple example of an abelian Lie group is the group $U(1)$ of complex numbers with absolute value 1 with multiplication among complex numbers as the group operation. The Lagrangean of QED, as given in Eq. (4), exhibits a local $U(1)$ symmetry.

In non-abelian gauge theories, the field tensor takes a more general form, as compared to Eq. (3), namely,

$$
F_{\mu \nu}^{a}=\partial_{\mu} A_{\nu}^{a}-\partial_{\nu} A_{\mu}^{a}+g f_{b c}^{a} A_{\mu}^{b} A_{\nu}^{c},
$$

in terms of which the Lagrangean describing the gauge fields can be written as

$$
\mathcal{L}=-\frac{1}{4} F_{\mu \nu}^{a} F^{\mu \nu a} .
$$

The different fundamental interactions between elementary particles are formulated in terms of different gauge groups. The gauge theory of the strong interaction, called chromodynamics, uses the symmetry group $S U(3)$. The matter particles between which it acts are called quarks, the gauge particles mediating it are called gluons. The weak and electromagnetic interactions are unified in a single gauge theory formulated in terms of a combination of the gauge groups $S U(2)$ and $U(1)$, written as $S U(2) \times U(1)$. The Higgs mechanism plays a crucial role in the standard account of why this interaction manifests itself in a twofold way, namely, on the one hand, in form of the long-range electromagnetic interaction and, on the other hand, in form of the short-range weak interaction. The combined electroweak interaction 
acts between all types of matter particles (both quarks and leptons, the latter of which include electrons and positrons) and is mediated by the photons and the gauge particles mediating the weak force, the weak gauge bosons, also known as the $W^{+}-, W^{-}$and $Z$-bosons.

\section{The mass problem and spontaneous symmetry breaking}

Historically, accounting for the strong and weak interactions in terms of gauge theories was a formidable challenge. In both cases, the main obstacle to a description of either of them in terms of a gauge theory was that, as determined experimentally, both the strong and the weak interaction appear to have a very short range, especially when compared to the electromagnetic interaction. Short-range interactions are mediated by particles having non-vanishing particle masses, but the Lagrangean Eq. (4) and its non-abelian generalisation such as Eq. (9) do not include mass terms for the gauge bosons, which would have the form (of being proportional to) $-m^{2} A_{\mu}^{a} A^{a \mu}$. Besides, simply adding mass terms $-m^{2} A_{\mu}^{a} A^{a \mu}$ for the gauge particles putatively mediating the strong and weak interactions raises problems in its own right, for it violates gauge invariance and prevents the theory from fulfilling the important requirement of renormalisability, meaning, roughly speaking, that the theory does not lose its predictivity when extrapolating it to very short distances and very large energy scales. Only mass terms $-m \bar{\psi} \psi$ for the matter particles are compatible with the local gauge symmetry. Interactions mediated by massless gauge bosons, however, are known to give rise to long-range interactions, for which the electromagnetic interaction provides a paradigmatic example. The slow $1 / r^{2}$-fall-off of the electrostatic force according to the Coulomb law and of the magnetostatic force according to the Biot-Savart law are prime examples of such long-range behaviour.

For the strong interaction, it became possible to solve the problem of how to describe it in terms of a gauge theory by realising that the particles between which it fundamentally acts are not the protons and neutrons forming the atomic nuclei but, instead, their sub-constituents known as the quarks. The gauge theory which accounts for the strong interaction is called quantum chromodynamics. As already remarked, the Lie group in terms of which it is formulated is $S U(3)$. As a binding force between quarks, the strong interaction turns out not to be a short-range force at all: rather than falling off, it grows indefinitely with increasing distance. In virtue of this feature 
it inevitably leads to the formation of bound states between quarks, the socalled mesons (consisting of two quarks) and hadrons (consisting of three), which are "colourless", that is, uncharged as far as the strong interaction is concerned. The constituents of atomic nuclei, the protons and neutrons, are examples of hadrons, and the resulting net-forces between these composite particles due to the strong interaction do indeed fall off rapidly, hiding the actual long-range character of the strong interaction and their growing with distance.

For the weak interaction, in contrast, no similar explanation of its shortrange character could be found, so one had to account by other means for the apparently massive character of the particles mediating it. The Higgs mechanism is the dominant paradigm for solving this problem, and the standard textbook account of how this is done will be reviewed in the following section. ${ }^{9}$

The notion of the spontaneous breaking of local gauge symmetry is widely regarded as the conceptual clue to the Higgs mechanism. This perspective, as will be argued in Section 5, is not completely adequate and should be replaced by a more complex picture. To understand it, however, a short review of the notion of spontaneous symmetry breaking is in order

The notion of spontaneous symmetry breaking, as it underlies that of spontaneous gauge symmetry breaking, is based on the insight that a symmetry of the laws which govern the behaviour of some physical system mayin a sense to be specified-be radically absent from its actual state. For a classical system, a symmetry of its laws of motion is spontaneously broken in a configuration if it is impossible to take the system to a symmetry-related configuration by means of "physically realizable operations." ${ }^{10}$ To see what this requirement amounts to in practice, consider a classical one-dimensional system with a double-well potential, as defined by $V(x)=\lambda\left(x^{2}-x_{0}^{2}\right)^{2}$ (with $\lambda>0$ ). The system is symmetric with respect to the transformation $x \mapsto-x$, but this symmetry is not spontaneously broken by any configuration of the system since the local maximum of the potential at $x=0$ has only finite height and in that sense can be overcome by "physically realizable operations." Under realistic conditions (that is, in the absence of potential

\footnotetext{
${ }^{9}$ The historically most significant contributions to the original development of the Higgs mechanism include [Anderson, 1963], [Englert and Brout, 1964], [Higgs, 1964], [Guralnik et al., 1964], [Kibble, 1967]. See [Karaca, 2012] for a recent critical study of the historical origins of the Higgs mechanism and the electroweak theory.

${ }^{10}$ See [Strocchi, 2008] p. 4. Strocchi's work gives a comprehensive and conceptually rigorous introduction to spontaneous symmetry breaking, which makes all of the notions employed here mathematically precise.
} 
barriers of infinite height), the system needs to have infinitely many degrees of freedom in order for its states to spontaneously break one of the symmetries of its laws of motion. Thus, the phenomenon of spontaneous symmetry breaking goes far beyond the rather trivial observation that solutions to symmetric equations of motions need not themselves by symmetric. In the quantum case, the basic idea of the concept of spontaneous symmetry breaking can be made precise in the language of the algebraic approach to quantum theories. For accessible introductions to this topic see [Ruetsche, 2011], Chapter 13, and [Strocchi, 2008], Part II.

Spontaneous symmetry is often illustrated in terms of the state of a magnetised solid material, where in the limit of an infinitely extended system the magnetisation axis "spontaneously breaks" the full rotational symmetry of the laws governing the behaviour of magnetic moments in the interior of the material. The expectation value of these magnetic moments is zero in the absence of rotational symmetry breaking and nonzero when rotational symmetry is broken. Quantities such as this expectation value are referred to as symmetry breaking order parameters. Typically, when they are zero in a pure state of the theory, the symmetry is unbroken; and when they are nonzero, it is broken.

In the case of the Higgs mechanism, the simplest and currently favoured approach is to postulate an additional type of physical particle - the "Higgs particle" that has now apparently been discovered-, which is claimed to be characterised by a non-vanishing vacuum expectation value of the field associated with it. Even though in this case the criterion for spontaneous symmetry breaking in terms of "physically realizable operations" does not literally apply (since different configurations related by gauge symmetry represent one and the same physical situation, which means that the question of whether one can be obtained from the other by means of physically realisable operations does not arise), the situation seems formally analogous to one of spontaneous symmetry breaking in the sense just discussed. This is the reason why - mistakenly, as I argue in Section 5-a nonzero vacuum expectation value of the Higgs field and an associated breaking of local gauge symmetry are widely taken to be at the origin of the generation of particle masses through the Higgs mechanism.

The way in which a non-vanishing vacuum expectation value of the Higgs field supposedly gives rise to non-vanishing masses for the gauge particles mediating the weak force is often illustrated by physicists in terms of analogies drawn from everyday life. Eminent CERN physicist John Ellis, for instance, reportedly illustrates it as follows: "Different fundamental particles [...] are like a crowd of people running through mud. Some particles, 
like quarks, have big boots that get covered with lots of mud; others, like electrons, have little shoes that barely gather any mud at all. Photons don't wear shoes - they just glide over the top of the mud without picking any up. And the Higgs field is the mud." ${ }^{11}$ In the following section, the perspective on the Higgs mechanism expressed in this quote is spelled out in a little more technical detail, though the discussion remains confined to the case of classical field theory. Section 5 considers the Higgs mechanism in the context of quantum field theory, where the simile used by Ellis (and similar similes used by many others) may seem less well applicable.

\section{The Higgs mechanism in classical field theory}

In this section, I discuss the textbook account of the Higgs mechanism in classical field theory in terms of the notion of a spontaneously broken local gauge symmetry. To see the underlying idea, it suffices to consider, as an example, the Lagrangean of the relatively simple abelian Higgs model. Here an abelian gauge field is coupled to a scalar field $\phi$ with a quartic "Mexican hat" type potential $V[\phi]=m_{0}^{2} \phi^{*} \phi+\lambda_{0}\left(\phi^{*} \phi\right)^{2}$ for which $m_{0}^{2}<0$ (which means that $m_{0}$ is a complex number with vanishing real part):

$$
\mathcal{L}=D_{\mu} \phi^{*} D^{\mu} \phi-V[\phi]-\frac{1}{4} F_{\mu \nu} F^{\mu \nu},
$$

where, as defined by Eq. (5), the covariant derivative $D_{\mu}=\partial_{\mu}+i e A_{\mu}$ encodes the coupling between $\phi$ and $A_{\mu}$.

This Lagrangean exhibits a local $U(1)$ gauge symmetry in that it is invariant under gauge transformations of the form

$$
\phi(x) \mapsto e^{i \alpha(x)} \phi(x), \quad A_{\mu}(x) \mapsto A_{\mu}(x)-\frac{1}{e} \partial_{\mu} \alpha(x) .
$$

Since the coefficient $m_{0}^{2}$ is assumed to be negative, the potential $V$ has minima at nonzero values of the Higgs field $\phi$, namely, those for which $|\phi|^{2}=-\frac{m_{0}^{2}}{2 \lambda_{0}}$.

The classical ground states of the theory are configurations of the fields $\phi$ and $A_{\mu}$ for which

$$
\phi(x)=e^{i \theta(x)} v / \sqrt{2}, \quad A_{\mu}(x)=-\frac{1}{e} \partial_{\mu} \theta(x),
$$

\footnotetext{
${ }^{11}$ See [Achenbach, 2008] p. 4.
} 
and $\theta(x)$ is an arbitrary real-valued function of space and time and $v=$ $\sqrt{-\frac{m_{0}^{2}}{\lambda_{0}}}$ corresponds to the value of the scalar field for which the potential $V$ has its minimum. For any two field configurations of the form Eq. (12) there exist gauge transformations of the form Eq. (11) that transform one into the other, so that, at least according to the standard view of gauge symmetries, all these configurations are physically equivalent and it makes no sense to claim for any of them that it is the one which is really chosen by the $\phi$-field. However, since $v \neq 0$, none of the field configurations (12) is itself invariant under local gauge transformations. This means that gauge field configurations minimising the classical energy of the theory defined by Eq. (10) are non-trivially mapped onto each other by gauge transformations in such a way that, at least formally, any ground state configuration spontaneously breaks local gauge symmetry. By this argument, the notion of a spontaneously broken local gauge symmetry is often regarded as the conceptual clue to the Higgs mechanism.

One objection to this standard account of the Higgs mechanism is to attack the claim that it involves a spontaneously broken gauge symmetry at all, since all of the symmetry breaking field configurations represent the same physical situation. This means that unlike in cases of "real" symmetry breaking - as in that of the magnetised material considered above - none of these field configuration is, as one might say, "picked out by nature" as the one that is actually realised. Perez and Sudarsky reject the notion of gauge symmetry breaking on just these grounds, arguing that "gauge degrees of freedom are not physical [in that] ... [t] hey are simply redundant fields that enter our mathematical description of certain physical interactions [so that,] [c]onsequently, a gauge symmetry cannot be broken not only by the vacuum, but by any state in the theory." 12 Indeed, given the nature of gauge symmetries as relating physically identical configurations and the criterion for symmetry breaking that requires the impossibility of taking a symmetry breaking configuration to a symmetry-related one by means of "physically realizable operations", this critique of the notion of gauge symmetry breaking is adequate.

However, it is equally possible and more in agreement with existing usage to accept the notion of gauge symmetry breaking and to apply it to those situations which are formally exactly analogous to those which exhibit symmetry breaking in the strict sense discussed in Section 2. If one chooses this route, as will be done in what follows, one has to keep in mind that gauge symmetry breaking differs crucially from symmetry breaking in cases of non-

\footnotetext{
${ }^{12}$ See Perez and Sudarsky [2008] p. 19
} 
gauge symmetries, where out of a multitude of physically distinct configurations (or states) only one, in contrast to all others, is actually realised. A distinction which continues to be salient in the case of gauge symmetries and which remains unproblematic is that between situations described in terms of gauge invariant field configurations and situations described by physically equivalent, yet mathematically distinct, field configurations which, each individually, formally break the local gauge symmetry. The classical minimum energy configurations of the theory defined through Eq. (10) are of the latter kind, and it is precisely in that sense that the spontaneous breaking of local gauge symmetry can be realised in a classical gauge theory.

Before discussing the status of spontaneous gauge symmetry breaking in quantum gauge theories, it is interesting to indicate how the theory defined by the Lagrangean (10) accounts for the appearance of non-vanishing masses for the gauge bosons. To start with, it is useful to perform the field redefinition

$$
\begin{aligned}
\phi(x)=e^{i \theta(x)} \rho(x) & \mapsto \rho(x), \\
A_{\mu}(x) & \mapsto A_{\mu}(x)+\frac{1}{e} \partial_{\mu} \theta(x) \equiv B_{\mu}(x),
\end{aligned}
$$

which makes it possible to eliminate the $\theta$-field from the Lagrangean, which thereby becomes

$$
\mathcal{L}=\partial_{\mu} \rho \partial^{\mu} \rho-V(\rho)+e^{2} \rho^{2} B_{\mu} B^{\mu}-\frac{1}{4} B_{\mu \nu} B^{\mu \nu},
$$

where $B_{\mu \nu} \equiv \partial_{\mu} B_{\nu}-\partial_{\nu} B_{\mu}$ has been defined.

Expanding the field $\rho$ around its expectation value as $\rho=(v+\eta) / \sqrt{2}$ and expanding up to second order in the fields $\eta$ and $B_{\mu}$ one obtains

$$
\mathcal{L}^{(2)}=\frac{1}{2}\left(\partial_{\mu} \eta \partial^{\mu} \eta+2 m_{0}^{2} \eta^{2}\right)+\frac{1}{2} e^{2} v^{2} B_{\mu} B^{\mu}-\frac{1}{4} B_{\mu \nu} B^{\mu \nu} .
$$

The characteristic physical properties of the theory defined by this Lagrangean can be read off by interpreting $M_{B}=e v$ and $\sqrt{-2 m_{0}^{2}}$ as the masses, respectively, of a vector boson associated with a vector field $B_{\mu}$ and a scalar boson associated with a real-valued scalar field $\eta$. The massive vector field fulfils the role of the desired massive field mediating a short-range force between matter fields (once they are coupled to the theory (10)), and the massive scalar field testifies to the presence of the original scalar field $\phi$, which has assumed a non-vanishing vacuum expectation value. The massless field $\theta$ has been eliminated as unphysical. This agrees nicely with the experimental evidence for the absence of massless scalar particles in elementary particle physics. 
In the part of the Standard Model which describes both the weak and the electromagnetic interaction, the implementation of the Higgs mechanism is slightly more complicated than just described in that, as already remarked, the local gauge symmetry of that theory is a non-abelian $S U(2) \times U(1)$ symmetry rather than the simpler abelian $U(1)$ symmetry of Eq. (10). Moreover, the $S U(2) \times U(1)$ symmetry is not completely broken by the Higgs field, but only up to a residual $U(1)$ symmetry, which coincides with the gauge symmetry of electromagnetism. Despite these important conceptual differences, the conclusion just established that the Higgs mechanism can be described as a case of a spontaneously broken local gauge symmetry is not affected and remains correct for the classical version of the electroweak theory.

It should be added here that in the fully-fledged Standard Model the Higgs particle is not only responsible for the masses of the gauge bosons mediating the weak interaction, but also for those of the matter particles such as quarks and electrons. The coupling to the Higgs particle thus accounts in an elegant and comprehensive way for the masses of all matter particles, including the massive weak gauge bosons. To translate these observations into the language of Ellis' simile, we may say that the non-vanishing amount of mud Ellis speaks of corresponds to a non-vanishing vacuum expectation value of the Higgs field, and the reduced mobility of the people moving through the mud corresponds to an increase in inertia of the particles that are coupled to the Higgs particle caused by the "stickiness" of the vacuum in the presence of a non-vanishing vacuum expectation value of the Higgs field.

Having discussed the Higgs mechanism in the context of classical field theory, I now turn to its status and interpretation in the context of quantised gauge theories.

\section{$5 \quad$ Quantisation and gauge symmetry breaking}

There exist various different frameworks for turning a classical theory into a quantum theory, often referred to as its "quantisation". For gauge theories, the framework of quantisation that is nowadays mostly used and most convenient is the Feynman functional integral method. Here, the classical action $S=\int d^{4} x \mathcal{L}$ occurs in the integrand of an integral over all possible field configurations, and the physical content of the quantised theory in form of the expectation values of observables is obtained from the integral by taking derivatives with respect to the fields. The central challenge when quantising a gauge theory by means of this approach arises from the fact 
that this integral ranges over each individual mathematically distinct field configuration and thereby takes into account each physically distinct field configuration infinitely often. This leads to serious problems when trying to set up the most-used (so-called "perturbative") computation methods to determine the expectation values of observables in the context of quantum field theories. There are two main distinct ways to deal with this problem, both of which have their characteristic merits and drawbacks.

The first is to try to extract physical results from the functional integral defining the quantised gauge theory differently than by means of the usual perturbative methods. The most prominent such approach is lattice quantisation, where one considers space-time not as a continuum but as a lattice of discrete space-time points and extrapolates the results obtained to the continuum case by letting the lattice spacing go to zero. In this approach, the local gauge symmetry used in the formulation of the classical theory to be quantised can be left untouched when quantising, and one can ask for any state of the theory whether it spontaneously breaks local gauge symmetry. It would do so just in case some gauge-dependent combination of fields were assigned a nonzero expectation value, which would then be able to serve as a symmetry breaking order parameter.

The second reaction to the problem encountered in functional integral quantisation of a gauge theory just described is to perform a change of integration variables in such a way that the integral is performed over only a restricted subclass of field configurations, taking into account each physically distinct field configuration much "less often" than in the original functional integral. One can then proceed to apply the standard perturbative methods of quantum field theory. This approach, which is known as "fixing the gauge", involves explicit violation of the invariance under local gauge transformations in the change of integration variables, so that after fixing the gauge there is no local gauge symmetry left to be broken. However, as will be discussed below, the question of a possible breakdown of global symmetries under which the theory may remain invariant after gauge fixing remains open. The approach that does not rely on gauge fixing is discussed first in what follows. 


\subsection{No local gauge symmetry breaking in gauge quantum field theory}

The most important result in this context is a rigorous result of lattice gauge theory, widely known as Elitzur's theorem. ${ }^{13}$ According to this result, local gauge symmetry cannot be spontaneously broken at all in a gauge quantum field theory in the sense that arbitrary gauge-dependent combinations (monomials) of fields must have zero expectation values. ${ }^{14}$ In particular, contrary to what seems to be suggested by similes such as that used by Ellis, the vacuum expectation value of the Higgs field itself has to be zero according to Elitzur's theorem.

The proof of the theorem is crucially based on the fact that there exist local gauge transformations which are different from the identity transformation only in a finite space-time region, i. e. which reduce to the identity transformation outside. This makes it impossible to impose a symmetry breaking order parameter by fixing it on the boundary of a finite space-time region and subsequently removing the boundary to infinity, which is one of the standard recipes for detecting spontaneous symmetry breaking. ${ }^{15}$

The theorem does not apply to theories which are formulated in terms of a global, rather than local, symmetry. For in the case of global symmetries, any non-trivial symmetry transformation must differ from the identity transformation in all of space-time; so that no symmetry transformations which are different from the identity transformation only in a finite spacetime region exist and the line of argument used to prove Elitzur's theorem cannot be run. Thus, the impossibility of breaking local gauge symmetries as stated by Elitzur's theorem is not a consequence of the general unobservability of gauge transformations but rather has to do with the specific features of local gauge symmetry transformations.

As a rigorous result Elitzur's theorem holds only in the framework of lattice gauge theory, so there remains the theoretical possibility that a future

\footnotetext{
${ }^{13}$ Elitzur proved the theorem for the case of a Higgs field with fixed modulus, see [Elitzur, 1975]. The result was generalised to the case of a Higgs field with variable modulus by de Angelis, de Falco and Guerra, see [De Angelis et al., 1978].

${ }^{14}$ More precisely, the theorem states that the expectation value of any monomial which transforms as a non-trivial irreducible representation of the local gauge group must vanish. I would like to thank an anonymous referee of this journal for proposing this concise formulation.

${ }^{15}$ See the proof sketches of Elitzur's theorem [Strocchi, 1985], Chapter II 2.5, and [Fröhlich et al., 1981], Section 3, for more details. For a more rigorous textbook version applied to the special case of the vacuum expectation value of the Higgs field see [Itzykson and Drouffe, 1989], Chapter 6.1.3.
} 
rigorous formulation of gauge quantum field theory in the space-time continuum may re-establish the notion of a spontaneously broken local gauge symmetry. However, since this notion is not supported by our currently best framework of gauge theory quantisation, namely, lattice quantisation, and the Standard Model of elementary particle physics is employed as a quantum, not classical, field theory, we have to conclude that to characterise the Higgs mechanism in the context of the Standard model as a spontaneously broken local gauge symmetry is misleading according to our currently best understanding of elementary particle physics.

It is important to add that this statement does not undermine the viability of the Higgs mechanism as an account of mass generation for the weak bosons that solves the problem of the short-range character of the weak force. As demonstrated by Fröhlich, Morchio, and Strocchi ${ }^{16}$, all the physical features which are usually associated with the Higgs mechanism can be described in terms of expectation values of only gauge-invariant combinations of fields. In particular, quantities corresponding to the mass terms of both the matter particles and the weak gauge bosons can be recovered as expectation values of gauge-invariant fields. So, local gauge symmetry breaking is not invoked by this account, and a non-vanishing expectation value is not assumed for any gauge-dependent combination of fields, in particular not for the Higgs field itself.

While local gauge symmetry itself cannot be spontaneously broken according to our currently best understanding of gauge quantum field theories, gauge symmetry breaking can occur after gauge fixing, in which case it is the so-called remnant global gauge symmetries which are potentially broken. In what follows I give a brief introduction to the notion of a remnant global gauge symmetry and clarify the relation between the breaking of these symmetries and the Higgs mechanism. ${ }^{17}$

\subsection{Remnant global gauge symmetry breaking}

For the discussion of remnant global gauge symmetry breaking I now turn to the second approach to the quantisation of gauge theories mentioned above, where one "fixes the gauge" and, doing so, violates local gauge invariance explicitly in the quantisation procedure. Fixing the gauge means to restrict the functional integral used to define the quantised gauge theory in such a

\footnotetext{
${ }^{16}$ See [Fröhlich et al., 1981].

${ }^{17}$ For more detailed discussions, see [Friederich, 2011] and [Caudy and Greensite, 2008]. The results presented in the latter paper provide the basis for the main claims made in what follows.
} 
way that it ranges only over field configurations which are compatible with some chosen constraint. A simple example is the unitary gauge, which fixes the phase of the Higgs field at a constant value, say zero, at any space-time point. For the Higgs field in the abelian Higgs model discussed in Section 4 , which can be written in terms of two real-valued fields $\theta(x)$ and $\phi(x)$ as $\phi(x)=e^{i \theta(x)} \rho(x)$, this means setting $\theta(x)=0$ for all $x$. Examples of other choices of gauge fixings, which are often better suited for practical purposes, include the Coulomb gauge, defined by $\partial_{i} A^{i}=0$ (where the summation is over spatial indices only), and the Lorenz gauge, defined by $\partial_{\mu} A^{\mu}=0$.

Gauge fixing constraints cannot be invariant under local gauge symmetry, for otherwise they could not fulfil their task of restricting the domain of field configurations where the functional integral receives non-vanishing contributions. If done correctly, however, gauge fixing violates local gauge invariance in such a way that the results concerning physical, that is, gaugeinvariant, quantities obtained from the functional integral do not depend on the choice of gauge fixing made. It is important to note that the way in which local gauge invariance is violated by gauge fixing depends on the details of one's choice of gauge fixing constraint. One possibility is that it eliminates the gauge freedom completely in the sense that out of any class of field configurations taken to each other by gauge transformations exactly one is singled out by the gauge fixing constraint. This is the case for the unitary gauge, which, as remarked, is given by $\theta(x)=0$ in the case of the locally $U(1)$-symmetric abelian Higgs model discussed above. In this case, the gauge symmetry is removed altogether by the constraint, so spontaneous gauge symmetry breaking cannot happen any more in that there is no unbroken gauge symmetry left to be broken. For other choices of gauge fixing, however, the gauge fixing constraint may be compatible with more than one out of each physically equivalent class of field configurations. In this case, the resulting theory (including the term which implements the gauge fixing constraint) is typically invariant under transformations corresponding to a global symmetry group. Since this symmetry group can be seen as a subgroup of the original local gauge group in the sense that the transformations which it contains form a subset of the full set of local gauge transformation, it has been called the subgroup of "remnant global gauge symmetries." 18 The present paper adopts this terminology.

There is now no result like Elitzur's theorem that forbids the spontaneous breaking of these symmetries ${ }^{19}$, and their breaking is indeed frequently found

\footnotetext{
${ }^{18}$ See [Caudy and Greensite, 2008].

${ }^{19}$ At first glance, the spontaneous breaking of remnant global symmetries may seem
} 
in many calculations based on gauge fixing. At this stage, it is certainly natural to ask whether the Higgs mechanism corresponds to the breaking of a remnant global symmetry, in which case the conventional wisdom of regarding symmetry breaking as the conceptual hallmark of the Higgs mechanism and the associated generation of particle masses might be vindicated inasmuch as remnant global symmetry breaking is involved.

The answer to this question is not completely straightforward, but arguably essentially negative. To begin with, for many choices of gauge fixing the resulting remnant global gauge symmetries are indeed spontaneously broken in regimes of the parameter space which display the typical features of the Higgs mechanism such as the generation of gauge boson masses. However, the distinction between broken and unbroken remnant gauge symmetry does not in general line up with an accompanying discontinuous change in (at least some) physical properties (meaning that it does not correspond to a transition between distinct physical phases), which means that remnant gauge symmetry breaking is not in general associated with any direct physical consequences. ${ }^{20}$

A further observation about remnant gauge symmetry breaking that suggests not to assign it a central role in the interpretation of the Higgs mechanism is that it is "ambiguous" (to use an expression borrowed from the title of [Caudy and Greensite, 2008]) in the sense that whether or not it occurs for a specific choice of parameters depends on the (from a physical point of view) arbitrary choice of gauge. To sum up with a general moral to be drawn from these considerations: even though in the presence of gauge fixing the typical features of the Higgs mechanism often occur together with the spontaneous breaking of some remnant subgroup, remnant gauge symmetry breaking cannot be taken as equivalent to, let alone causally responsible for, the generation of particle masses through the Higgs mechanism.

The considerations concerning Elitzur's theorem and remnant gauge symmetry breaking just presented have interesting ramifications for the de-

astonishing in the light of Elitzur's theorem, since global symmetry transformations are special cases of local symmetry transformations, and local gauge symmetries cannot break spontaneously, as Elitzur's theorem assures us. The puzzle is resolved by noting that the global symmetries are potentially broken only in the gauge fixed theory, where local gauge symmetry is no longer present. One should note, however, that results about remnant gauge symmetry breaking can be recovered by lattice calculations without gauge fixing in a more indirect manner as well, which means that, from a methodological point of view, gauge fixing is not the only option to determine remnant global gauge symmetry breaking.

${ }^{20}$ See [Caudy and Greensite, 2008] for a detailed elaboration and defence of these claims, based on detailed numerical investigations as well as on an earlier rigorous result due to Fradkin and Shenker [1979]. 
bates about the "reality" of gauge symmetries that were briefly touched upon in the introduction to gauge symmetries in Section 2. Thus, Nobel laureate Steven Weinberg once argued that gauge symmetries must be regarded as real in a substantive sense, given the (theoretically predicted) restoration of broken (remnant) gauge symmetry at high temperature. He claims: "if a gauge symmetry becomes unbroken for sufficiently high temperature, it becomes difficult to doubt its reality." ${ }^{21}$ The main idea behind Weinberg's claim seems to be that if the contrast between qualitatively different physical phases (between which transitions occur at very high temperature) can be shown to correspond to a distinction between broken or unbroken gauge symmetry, the symmetry must be the bearer of non-trivial physical properties and, therefore, must be real. Although it may be debated in which sense gauge symmetries are supposedly established as "real" according to this line of thought, the considerations presented earlier in this section seem to suggest that Weinberg's argument fails, whatever exactly it is meant to show.

For the notion of a spontaneously broken local gauge symmetry is, as we know from Elitzur's theorem, not supported by our present-day knowledge of gauge quantum field theory. So there is no distinction between broken and unbroken local gauge symmetry available to support Weinberg's argument. Remnant global gauge symmetries, in contrast, may indeed break spontaneously (and Weinberg grounds his claim on an example of a remnant global symmetry); and for some choices of gauge fixing and of remnant global subgroup, the distinction between broken and unbroken remnant global gauge symmetry may indeed line up with a distinction between different phases. However, as just discussed, remnant gauge symmetry breaking is ambiguous in that for different choices of gauge fixing, remnant gauge symmetry may occur at different values in parameter space (or not at all). This means that remnant gauge symmetry breaking does not in general correspond to a contrast between qualitatively different phases, as the line of thought suggested by the quotation from Weinberg suggests. We are therefore given no reason to regard these global symmetries as the true bearers of physical properties and as "real" in any more substantive sense than the local symmetries, which are used to formulate the gauge theories. While Weinberg's argument in favour of the "reality" of gauge symmetries along these lines fails, this does not seem to speak against accounts of gauge symmetries as real in a more indirect sense such as in Lyre's structural realist account mentioned in Section 2. However, neither gauge symmetry breaking nor the

${ }^{21}$ See [Weinberg, 1974] p. 3359. 
Higgs mechanism seem to support a more "robustly realist" interpretation of gauge symmetries than the standard account of gauge transformations as connecting physically identical states of affairs.

\section{Concluding remark}

The paper has discussed the relation between the Higgs mechanism and the notion of a spontaneously broken gauge symmetry. After introducing the basic notions of gauge theories, the motivation for the Higgs mechanism was reviewed by outlining the problem of how to account for the experimentally manifest short-range character of the weak force in terms of a gauge theory. Subsequently, the standard textbook account of the Higgs mechanism as a spontaneously broken local gauge symmetry in classical field theory was reviewed by illustrating how minimal energy configurations of the gauge fields may violate local gauge invariance. Finally, it was emphasised that according to our currently best understanding of gauge quantum theories, local gauge symmetry itself cannot be spontaneously broken (Elitzur's theorem), whereas remnant global subgroups of the original local gauge group may indeed break spontaneously. However, since the breaking of these latter symmetries does not in general correspond to a qualitative change in physical properties and depends on the choice of gauge, which is - from a physical point of view - arbitrary, the notion of remnant global gauge symmetry breaking should, I have argued, not be seen as the conceptual clue to the generation of particle masses through the Higgs mechanism.

\section{Acknowledgements}

I would like to thank Jeremy Butterfield, Friedrich Harbach, Koray Karaca and two anonymous referees for many helpful comments on earlier versions of this paper.

\section{References}

Achenbach, J., At the Heart of All Matter: The Hunt for the God Particle, National Geographic, available online at http://ngm.nationalgeographic.com/2008/03/god-particle/ achenbach-text (2008), 
Anderson, P. W., Plasmons, gauge invariance and mass, Physical Review 130:439-442 (1963),

Caudy, W. and Greensite, J., Ambiguity of spontaneously broken gauge symmetry, Physical Review D 78:025018 (2008),

De Angelis, G. F., De Falco, D., and Guerra, F., Note on the abelian HiggsKibble model on a lattice: Absence of spontaneous magnetization, Physical Review D 17:1624-28 (1978),

Earman, J., Laws, symmetry, and symmetry breaking: Invariance, conservation principles, and objectivity, Philosophy of Science 71:1227-41 (2004),

Elitzur, S., Impossibility of spontaneously breaking local symmetries, Physical Review D 12:3978-82 (1975),

Englert, F. and Brout, R., Broken symmetry and the mass of gauge vector mesons. Physical Review Letters 13:321-323 (1964),

Fradkin, E. and Shenker, S. H., Phase diagrams of lattice gauge theories with Higgs fields, Physical Review D 19:3628-97 (1979),

Friederich, S., Gauge symmetry breaking in gauge theories, preprint, available at http://philsci-archive.pitt.edu/8854/ (2011),

Fröhlich, J., Morchio, G., and Strocchi, F., Higgs phenomenon without symmetry breaking order parameter, Nuclear Physics B 190:553-82 (1981),

Greaves, H. and Wallace, D., Empiricial consequences of symmetries, preprint, available at http://philsci-archive.pitt.edu/8906/ (2011),

Greensite, J., An Introduction to the Confinement Problem, Springer: Berlin (2011),

Guralnik, G. S., Hagen, C. R., and Kibble, T. W. B. (1964). Global conservation laws and massless particles. Phys. Rev. Lett. 13: 585-7.

Healey, R., Quantum analogies: A reply to Maudlin, Philosophy of Science 66:440-447 (1998),

Healey, R., Gauging what's real: The conceptual foundations of contemporary gauge theories, Oxford University Press: New York (2007),

Healey, R., Perfect symmetries, British Journal for the Philosophy of Science 60:697-720 (2009), 
Higgs, P. W., Broken symmetries and the masses of gauge bosons. Physical Review Letters 13:508-509 (1964),

Itzykson, C. and Drouffe, J.-M., Statistical Field Theory. Vol.1: From Brownian motion to renormalization and lattice gauge theory, Cambridge University Press: Cambridge (1989),

Jackson, J. D., Classical Electrodynamics, 3rd edition, Wiley: New York (1998),

Karaca, K., The construction of the Higgs mechanism and the emergence of the electroweak theory, forthcoming in Studies in History and Philosophy of Modern Physics (2012),

Kibble, T. W. B., Symmetry breaking in non-abelian gauge theories, Physical Review 155:1554-1561 (1967),

Lyre, H., Humean perspectives on structural realism, in: Stadler, F. (ed.) The present situation in the philosophy of science, Springer Netherland: Berlin, pp. 381-397 (2010),

Maudlin, T., Healey on the Aharonov-Bohm effect, Philosophy of Science 65:361-368 (1998),

Noether, E., Invariante Variationsprobleme, Nachrichten der königlichen Gesellschaft der Wissenschaften zu Göttingen, Mathematischphysikalische Klasse Book 2:235-57 (1918), English translation by M. A. Tavel available at http://arxiv.org/abs/physics/0503066v1,

Ruetsche, L., Interpreting Quantum Theories, Oxford University Press: Oxford (2011),

Perez, A., Sudarsky, D., On the symmetry of the vacuum in theories with spontaneous symmetry breaking, http://arxiv.org/abs/0811.3181,

Roberts, B. W., Group structural realism, The British Journal for the Philosophy of Science, 62:47-69 (2011),

Smeenk, C., The elusive Higgs mechanism, Philosophy of Science 73:487-99 (2006),

Strocchi, F., Elements of Quantum Mechanics of Infinite Systems, World Scientific: Singapore (1985), 
Strocchi, F., Symmetry Breaking, 2nd edition, Springer: Berlin, Heidelberg (2008),

Struyve, W., Gauge invariant accounts of the Higgs mechanism, Studies in History and Philosophy of Modern Physics 42:226-236 (2011),

Weinberg, S., Gauge and global symmetry at high temperature, Physical Review D 9:3357-78 (1974). 